\title{
OSCILLATION, CONTINUATION, AND UNIQUENESS OF SOLUTIONS OF RETARDED DIFFERENTIAL EQUATIONS
}

\author{
BY \\ T. BURTON AND R. GRIMMER
}

\begin{abstract}
In this paper we present a number of results on continuation and uniqueriess of solutions of the $n$-dimensional system $x^{\prime}(t)=f(t, x(t))+g(t, x(t-\tau(t)))$ for $\tau(t) \geq 0$. We then give some necessary, some sufficient, and some necessary and sufficient conditions for oscillation of solutions of the second order equation $x^{\prime \prime}+a(t) f(x(t-\tau(t)))$ $=0$.
\end{abstract}

1. Introduction. During the last several years a number of exceptionally sharp results regarding uniqueness, continuation, and oscillation of solutions of the nonlinear second order differential equation

$$
x^{\prime \prime}+a(t) f(x)=0
$$

have been obtained. Atkinson [1] gave necessary and sufficient conditions for all solutions of $(\mathrm{N})$ to oscillate when $a(t)>0$ and $f(x)=x^{2 n+1}$ for $n \geq 1$. That result was extended by Gollwitzer [11] to

$$
x^{\prime \prime}+a(t) f(x(t-\tau(t)))=0
$$

for $a(t)>0, \tau(t)$ positive and bounded, and $f(x)=x^{\gamma}$ where $\gamma$ is the ratio of odd positive integers and $\gamma \neq 1$. An example by Waltman [16] can be modified to show that Gollwitzer's result will fail if $\tau(t)$ is allowed to become unbounded.

The present authors have investigated $(\mathrm{N})$ under the assumptions that $x f(x)$ $>0$ for $x \neq 0, f$ continuous, and (usually) $a(t)$ differentiable. We gave necessary and sufficient conditions for:

(a) continuation of all solutions of $(\mathrm{N})$ when $a(t)$ becomes negative at a point [5],

(b) uniqueness of the zero solution of $(\mathrm{N})$ when $a(t)$ becomes negative at a point [6], and

(c) oscillation of all solutions of $(\mathrm{N})$ when $a(t)$ is monotone increasing and $a(t)>0$ [3] and [4].

This collection of results gives one a fairly clear picture of many of the fundamental properties of solutions of second order equations of that general type. To date, there are mainly only isolated and specialized results on the specific nature of solutions of (D).

Received by the editors October 28, 1971 and, in revised form, May 10, 1972.

AMS (MOS) subject classifications (1970). Primary 34K05, 34K15, 34K99.

Key words and phrases. Oscillation, continuation, uniqueness. 
In this paper we explore the possibility of extending the results of $(N)$ to (D) and show that this is usually possible. The results on uniqueness and continuation extend as sufficient and necessary conditions respectively. One additional improvement is made in the form of a necessary and sufficient condition for oscillation of all solutions of (D) when $a(t)>0$ and $f(x)$ is monotone decreasing for $|x|$ large. This condition also holds when $\tau(t)=0$, thereby providing a new oscillation criterion for $(\mathrm{N})$.

Preparatory to our work with (D), we obtain several preliminary results on uniqueness and continuation of solutions of general $n$-dimensional systems.

A general discussion of existence for the initial value problem may be found in Chapter 2 of [14].

2. Systems of higher order. We first consider the system of retarded differential equations

$$
x^{\prime}(t)=u(t, x(t))+q(t, x(t-\tau(t))), \quad{ }^{\prime}=d / d t,
$$

anci the associated system of unretarded equations,

$$
y^{\prime}(t)=u(t, y(t))+p(t)
$$

in which $u, q, p$, and $\tau$ are continuous functions satisfying the following conditions:

(a) $u:[0, \infty) \times R^{n} \rightarrow R^{n}$.

(b) $q:[0, \infty) \times R^{n} \rightarrow R^{n}$.

(c) $p:[0, \infty) \rightarrow R^{n}$.

(d) $\tau:[0, \infty) \rightarrow[0, \infty)$.

Theorem 1. Suppose that $\tau(t)>0$ for all $t$ in $[0, \infty)$ and suppose that every solution of (1)' defined at any $t_{0} \geq 0$ can be continued as a solution of $(1)^{\prime}$ on $\left[t_{0}, \infty\right)$ for every continuous $p(t)$. If $x(t)$ is any solution of (1) defined on an interval $0 \leq t_{0} \leq t<t_{1}$ having continuous initial condition on $E_{t_{0}}=\left\{s \mid s=t-\tau(t) \leq t_{0}\right\}$ $\cup\left\{t_{0}\right\}$, then $x(t)$ can be continued as a solution past $t_{1}$.

Proof. As $x(t)$ is a solution having continuous initial condition and, as $\tau(t)$ is continuous and positive, $x(t-\tau(t))$ is continuous on the closed interval $\left[t_{0}, t_{1}\right]$. Thus, the function $p$ defined by $p(t)=q(t, x(t-\tau(t)))$ is continuous on the closed interval $\left[t_{0}, t_{1}\right]$ and can be extended to a continuous function on $[0, \infty)$. With $p$ defined in this manner, any solution $y(t)$ of $(1)^{\prime}$ satisfying $y\left(t_{0}\right)=x\left(t_{0}\right)$ can be continued as a solution of (1)' past $t_{1}$. But $x(t)$ is a solution of $(1)^{\prime}$ on $\left[t_{0}, t_{1}\right)$ and hence can be continued to $t_{1}$. That is, $\lim _{t \rightarrow t_{1}}\|x(t)\|$ exists as a finite number. According to Driver [9] (cf. also [14, p. 29]), the only way in which $x(t)$ can fail to be defined past $t_{1}$ is for $\lim \sup _{t \rightarrow t_{1}^{-}}\|x(t)\|=+\infty$. As this limit is finite for $x(t)$, the proof is complete. 
For many special equations such as the Liénard equation $x^{\prime \prime}+\phi\left(x, x^{\prime}\right) x^{\prime}$ $+\eta(x)=p(t)$ it is known [7] that all solutions are continuable to $+\infty$ whenever $\phi, \eta$, and $p$ are continuous, $\phi \geq 0$, and $x \eta(x)>0$ for $x \neq 0$. Hence, solutions are still continuable when terms $g\left(t, x(t-\tau(t)), x^{\prime}(t-\tau(t))\right)$ are added. On the other hand, the following result yields a continuation criterion for a wide class of problems.

Corollary. Suppose that $\tau(t)>0$ and that there are continuous functions $W$ and $r, W:[0, \infty) \rightarrow(1, \infty)$ and $r:[0, \infty) \rightarrow[0, \infty)$, such that $\|u(t, x)\| \leq r(t) W(\|x\|)$ and $\int_{0}^{\infty}(d v / W(v))=+\infty$. Then the conclusion of Theorem 1 holds.

Proof. Define $t_{0}, t_{1}$, and $p(t)$ as in the proof of the theorem. Then define $P=\max _{t_{0} \leq t \leq t_{1}}\|p(t)\|, \bar{r}(t)=r(t)+1$, and $\bar{W}(v)=W(v)+P$. Thus, for $t \geq t_{0}$, we have $\|u(t, y)+p(t)\| \leq r(t) W(\|y\|)+P \leq \bar{r}(t) \bar{W}(\|y\|)$. Now $\int_{0}^{\infty}(d v / W(v))=\infty$ implies that $\int_{0}^{\infty}(d v /(W(v)+P))=\infty$. Thus, it follows [12] that all solutions of (1)' can be continued to $+\infty$.

Remark. For ordinary differential equations without a time lag, say system (1)', the only way in which a solution $y(t)$ defined on $\left[t_{0}, t_{1}\right)$ can fail to be defined past $t_{1}$ is for $\lim _{t \rightarrow t_{1}^{-}}\|y(t)\|=+\infty$ (cf. [8]). This is a very important and useful property and is one which is not generally shared by solutions of (1) whose noncontinuable solutions merely satisfy $\lim \sup _{t \rightarrow t_{1}^{-}}\|x(t)\|=+\infty$. The following result gives sufficient conditions for the stronger property to hold for (1).

Theorem 2. Suppose that $\|u(t, x)\| \leq h(t)\|x\|$ for all $(t, x) \in[0, \infty) \times R^{n}$ and some continuous function $h:[0, \infty) \rightarrow[0, \infty)$. Let $x(t)$ be a solution of $(1)$ defined on $\left[t_{0}, t_{1}\right)$ and having continuous initial condition. If $x(t)$ cannot be defined past $t_{1}$, then $\lim _{t \rightarrow t_{1}^{-}}\|x(t)\|=+\infty$.

Proof. We first show that for any sequence $\left\{t_{n}\right\}$ tending strictly upward to $t_{1}$, we can find a sequence $\left\{t_{n}^{*}\right\}$ tending strictly upward to $t_{1}$ with $t_{n}=t_{n}^{*}-\tau\left(t_{n}^{*}\right)$.

Let $t_{n}>t_{0}$ and $\left\{t_{n}\right\}$ tend to $t_{1}$. As $\tau(t) \geq 0, t-\tau(t) \leq t$ and so $t_{0}-\tau\left(t_{0}\right) \leq t_{0}$ $<t_{n}$. Also, $t_{1}-\tau\left(t_{1}\right)=t_{1}>t_{n}$ since $\tau\left(t_{1}\right)=0$. (If $\tau\left(t_{1}\right)$ were positive, then solutions would be continuable past $t_{1}$.) Therefore, by the intermediate value theorem there exists $s$ satisfying $t_{0}<s<t_{1}$ with $s-\tau(s)=t_{n}$. Thus, if $A_{n}$ $=\left\{t \mid t_{0} \leq t \leq t_{1}, t-\tau(t)=t_{n}\right\}$, then $A_{n}$ is bounded, closed, and nonempty. Define $t_{n}^{*}=\sup A_{n}$ so that $t_{n}^{*}-\tau\left(t_{n}^{*}\right)=t_{n}$. We now show that $\left\{t_{n}^{*}\right\}$ is monotone and $t_{n}^{*}$ is strictly increasing to $t_{1}$. If $\left\{t_{n}^{*}\right\}$ is not increasing, then there exists $n$ with $t_{n}^{*} \geq t_{n+1}^{*}$. Then $t_{n}^{*}-\tau\left(t_{n}^{*}\right)=t_{n}<t_{n+1}=t_{n+1}^{*}-\tau\left(t_{n+1}^{*}\right)$. Also, as $t_{n}^{*} \geq t_{n+1}^{*}$, we must have $t-\tau(t)<t_{n+1}$ for $t$ in $\left(t_{n}^{*}, t_{1}\right]$. (If not, then there exists $t_{n+1}^{* *}$ in $\left(t_{n}^{*}, t_{1}\right] \subset\left(t_{n+1}^{*}, t_{1}\right]$ such that $t_{n+1}^{* *}-\tau\left(t_{n+1}^{* *}\right)=t_{n+1}$ which is a contradiction of our choice of $t_{n+1}^{*}$.) This says, however, that $t_{1}=t_{1}-\tau\left(t_{1}\right)<t_{n+1}<t_{1}$, which is a contradiction. It is clear that $\left\{t_{n}^{*}\right\}$ converges to $t_{1}$ as $t_{1}-\tau\left(t_{1}\right)=t_{1}$ and $\tau$ is continuous. 
If $\lim _{t \rightarrow t_{1}^{-}}\|x(t)\| \neq+\infty$, but lim $\sup _{t \rightarrow t_{1}^{-}}\|x(t)\|=+\infty$, then there exists $M>0$ and sequences $\left\{t_{n}\right\},\left\{\bar{t}_{n}\right\}$ strictly increasing to $t_{1}$ with $M \leq\|x(t)\| \leq 2 M$ for $t_{n} \leq t \leq \bar{t}_{n}$ and $\left\|x\left(t_{n}\right)\right\|=M,\left\|x\left(\bar{t}_{n}\right)\right\|=2 M$. Thus, we can find a sequence $\left\{t_{n}^{*}\right\}$ increasing to $t_{1}$ with $t_{n}=t_{n}^{*}-\tau\left(t_{n}^{*}\right)$. Then $\left\|x\left(t_{n}^{*}-\tau\left(t_{n}^{*}\right)\right)\right\|=M$ and there are sequences $\left\{s_{n}\right\}$ and $\left\{\bar{s}_{n}\right\}$ strictly increasing to $t_{1}$ with $M \leq\|x(t-\tau(t))\| \leq 2 M$ for $s_{n} \leq t \leq \bar{s}_{n}, M=\left\|x\left(s_{n}-\tau\left(s_{n}\right)\right)\right\|$, and $2 M=\left\|x\left(\bar{s}_{n}-\tau\left(\bar{s}_{n}\right)\right)\right\|$. Thus, $s_{n} \leq s \leq \bar{s}_{n}$ implies that

$$
\|x(s)\| \leq\left\|x\left(s_{n}\right)\right\|+\int_{s_{n}}^{s}\|u(t, x(t))\| d t+\int_{s_{n}}^{s}\|q(t, x(t-\tau(t)))\| d t
$$

and there is a number $Q>0$ with $\|q(t, x(t-\tau(t)))\| \leq Q$ on that interval. Hence, by Gronwall's inequality, $\|x(s)\| \leq\left(\left\|x\left(s_{n}\right)\right\|+Q\left(\bar{s}_{n}-s_{n}\right)\right) \exp \int_{s_{n}}^{s} h(t) d t$. Now the only way in which $t_{1}$ can be finite is for $\bar{s}_{n}-s_{n} \rightarrow 0$ as $n \rightarrow \infty$. Thus, $\|x(s)\|$ approaches $\left\|x\left(s_{n}\right)\right\|$ as $n \rightarrow \infty$. This, however, contradicts $\left\|x\left(\bar{s}_{n}\right)\right\|$ $=2\left\|x\left(s_{n}\right)\right\|$.

The problem of uniqueness of solutions of (1) is similar to that of continuability of solutions. The next result shows that if $u(t, x)$ is fairly smooth and if $\tau(t)>0$ then solutions of (1) are uniquely determined by their initial functions, independent of the lack of smoothness of $g$ (aside from continuity).

Theorem 3. Suppose that $\tau(t)>0$ for $0 \leq t<\infty$ and suppose that for every continuous function $p$, all solutions of (1)' are uniquely determined by their initial conditions. If $x(t)$ and $z(t)$ are two solutions of (1) defined on some interval $0 \leq t_{0} \leq t \leq t_{1}$ having the same continuous initial function $\phi$ on the initial interval $E_{t_{0}}=\left\{t_{0}\right\} \cup\left\{s \mid s=t-\tau(t)<t_{0}\right.$ for $\left.t \geq t_{0}\right\}$, then $x(t) \equiv z(t)$ for $t_{0} \leq t \leq t_{1}$.

Proof. As $\tau(t)>0$ on $t_{0} \leq t \leq t_{1}$, there exists $T>0$ with $\tau(t) \geq T$ on that interval. Also, if $x(t) \not \equiv z(t)$, then there is a number $t_{2}$ satisfying $t_{0}<t_{2} \leq t_{1}$ with $x\left(t_{2}\right) \neq z\left(t_{2}\right)$, but $x(t) \equiv z(t)$ for $t_{0} \leq t \leq t_{2}-T$. That is, the length of $E_{t_{0}}$ is at least $T$ and so for $t_{0} \leq t \leq t_{0}+T$, any solution $x_{\phi}(t)$ of (1) with initial function $\phi$ is a solution of $y^{\prime}=u(t, y)+g(t, \phi(t))$ whose solutions are uniquely determined by the initial point. Therefore, $\left[t_{0}, t_{2}-T\right]$ contains at least one point. Now, for $t_{0} \leq t \leq t_{2}$, we have $x(t)$ and $z(t)$ both being solutions of (1)' with $p(t)$ $=q(t, x(t-\tau(t)))$. As $x\left(t_{0}\right)=z\left(t_{0}\right)$ and solutions of $(1)^{\prime}$ are unique, it follows that $x(t)=z(t)$ for $t_{0} \leq t \leq t_{2}$. This completes the proof.

Corollary. Let $\tau(t)>0$ and let $u$ satisfy a local Lipschitz condition in $x$. Then the conclusion of the theorem holds.

3. Second order systems. We now turn to the system of two first order differential equations with time delay

$$
x^{\prime}(t)=y(t), \quad y^{\prime}(t)=-a(t) f(x(t-\tau(t)))
$$


in which $a(t), f$, and $\tau$ are continuous functions satisfying

(d) $\tau:[0, \infty) \rightarrow[0, \infty)$ and $t-\tau(t) \rightarrow \infty$ as $t \rightarrow \infty$,

(e) $a:[0, \infty) \rightarrow(-\infty, \infty)$ and

(f) $f:(-\infty, \infty) \rightarrow(-\infty, \infty)$ and $x f(x)>0$ if $x \neq 0$.

Our Theorems 1, 2, and 3 apply to system (2) in obvious ways.

When $\tau(t)=0$, then there are two ways in which a solution $(x(t), y(t))$ defined on an interval $\left[t_{0}, t_{1}\right)$ can fail to be defined past $t_{1}$. If $a\left(t_{1}\right)<0$, then we may have $\lim _{t \rightarrow t_{1}^{-}}|x(t)|=+\infty$. On the other hand, if $a\left(t_{1}\right)>0$ and if $a(t)$ is not of bounded variation, then $x(t)$ may oscillate unboundedly and $\lim _{t \rightarrow t_{1}^{-}}(|x(t)|+|y(t)|)=+\infty$ (cf. [13]). In [5] we derived a necessary and sufficient criterion for the first type of behavior. Here, we show that the same condition is a valid necessary condition when $\tau(t) \geq 0$.

Theorem 4. Let $a(t)<0$ on an interval $0 \leq t_{0} \leq t \leq t_{1}$. Suppose also that $f$ is increasing. Then there is a solution $(x(t), y(t))$ of $(2)$ defined on $\left[t_{0}, t_{1}\right)$ and satisfying $\lim _{t \rightarrow t_{1}}|x(t)|=+\infty$ only if either

$$
\int_{0}^{\infty} \frac{d x}{\sqrt{1+F(x)}}<\infty
$$

or

$$
\int_{0}^{-\infty} \frac{d x}{\sqrt{1+F(x)}}>-\infty
$$

where

$$
F(x)=\int_{0}^{x} f(s) d s
$$

Proof. We assume that there is a solution $X(t)=(x(t), y(t))$ defined on $\left[t_{0}, t_{1}\right)$ with $\lim _{t \rightarrow t_{1}^{-}} x(t)=+\infty$. Clearly, this must happen in Quadrant I of the $x y$-plane since $x^{\prime}(t)=y(t)$. Also, by the proof of Theorem 1, it must be the case that $\tau\left(t_{1}\right)=0$; otherwise, there exists an $\varepsilon>0$ and $\delta>0$ such that $\tau(t) \geq \varepsilon$ on $t_{1}-\delta \leq t \leq t_{1}+\delta$ and so $X(t)$ could be continued past $t_{1}$.

Now let $0<m<M$ be chosen so that $-M \leq a(t) \leq-m$ for $t_{0} \leq t \leq t_{1}$. Then $y y^{\prime}=-a(t) f(x(t-\tau(t))) y$ and since $\tau\left(t_{1}\right)=0, t_{1}>0$, we see that $t-\tau(t)$ $\rightarrow t_{1}$ as $t \rightarrow t_{1}$. Since $x(t) \rightarrow \infty$ as $t \rightarrow t_{1}$, there exists $t_{2}$ satisfying $t_{0} \leq t_{2}<t_{1}$ with $x(t-\tau(t))>0$ if $t_{2} \leq t<t_{1}$. Thus, $t \geq t_{2}$ implies that

$$
y y^{\prime}=-a(t) f(x(t-\tau(t))) y \leq-a(t) f(x(t)) y \leq M f(x(t)) x^{\prime}(t)
$$

and hence,

$$
y^{2}(t) \leq y^{2}\left(t_{2}\right)+2 M\left[F(x(t))-F\left(x\left(t_{2}\right)\right)\right]
$$


follows from an integration from $t_{2}$ to $t$. Therefore,

$$
x^{\prime}(t) /\left[y^{2}\left(t_{2}\right)+2 M\left\{F(x(t))-F\left(x\left(t_{2}\right)\right)\right\}\right]^{1 / 2} d x \leq d t
$$

or

$$
\int_{x\left(t_{2}\right)}^{x(t)}\left[y^{2}\left(t_{2}\right)+2 M\left\{F(x(t))-F\left(x\left(t_{2}\right)\right)\right\}\right]^{-1 / 2} d t \leq t-t_{2} .
$$

As $x(t) \rightarrow \infty$ when $t \rightarrow t_{1}^{-}>t_{2}$, it follows that (a) holds.

A similar argument holds in Quadrant III for (b) if $x(t) \rightarrow-\infty$ as $t \rightarrow t_{1}^{-}$. That argument will complete the proof.

Remark. The above proof is a straightforward adaptation of the proof given by the authors [5] for the equation without retardation. Although the condition does not appear to be both necessary and sufficient for delay equations, the equation

$$
x^{\prime \prime}-(12)(3 / 2)^{5} x^{5 / 3}\left(t-\left(1-\frac{1}{2} t\right)\right)=0
$$

has $x(t)=(t-2)^{-3}$ as a solution. Thus, there are equations satisfying (a) and (b) which have solutions with finite escape time.

In the same manner, if $\tau(t)>0$, then solutions are unique. However, for $t_{0}=0$ in the equation

$$
x^{\prime \prime}-12 x^{1 / 3}(t / 2)=0
$$

the initial interval is the single point $t_{0}$ and there are two solutions $x(t)=t^{3}$ and $x(t) \equiv 0$ satisfying $x(0)=x^{\prime}(0)=0$.

The following result is also patterned from our previous work [6] on unretarded equations. Again, the condition given does not appear to be both a necessary and sufficient condition for (2), although it is for the unretarded counterpart of (2).

Theorem 5. Suppose that there exists $t_{0} \geq 0$ and $t_{1}>t_{0}$ for which $\tau\left(t_{0}\right)=0$, $t_{0}<t-\tau(t) \leq t_{1}$ for $t_{0}<t \leq t_{1}$, and $a(t)<0$ on $\left[t_{0}, t_{1}\right]$. Finally, let $f$ be increasing. If

(i) $\int_{0^{+}}^{1}[F(x)]^{-1 / 2} d x=\infty$ and

(ii) $\int_{0}^{-1}[F(x)]^{-1 / 2} d x=-\infty$, then the zero solution of (2) is unique to the right at $t_{0}$.

Proof. Notice that the initial interval is just a point, $t_{0}$. Suppose (i) and (ii) hold but there exists a solution of (2), say $(x(t), y(t))$, which is zero on the initial interval, but not identically zero on $\left[t_{0}, t_{1}\right]$.

From the direction field defined by (2), it follows that $(x(t), y(t))$ is in the (closed) first or third quadrant for $t_{0} \leq t \leq t_{1}$. We shall assume that it is in the first quadrant.

As $a(t)$ is continuous, there exist $M$ and $m$ with $m<a(t)<M<0$ for $t_{0} \leq t \leq t_{1}$. Then for $t_{0} \leq t \leq t_{1}$, we have from (2) that 


$$
y y^{\prime}=-a(t) f(x(t-\tau(t))) x^{\prime}(t) \leq-a(t) f(x(t)) x^{\prime}(t)
$$

as $x(t)$ is increasing and $t-\tau(t) \leq t$. Thus, for $t_{0} \leq t_{2} \leq t \leq t_{1}$, we obtain upon integration

$$
\begin{aligned}
y^{2}(t)-y^{2}\left(t_{2}\right) & \leq-2 m \int_{t_{2}}^{t} f(x(s)) x^{\prime}(s) d s \\
& =-2 m F(x(t))+2 m F\left(x\left(t_{2}\right)\right) \leq-2 m F(x(t))
\end{aligned}
$$

and so $y(t) \leq \sqrt{y^{2}\left(t_{2}\right)-2 m F(x(t))}$ or

$$
x^{\prime}(t)\left[y^{2}\left(t_{2}\right)-2 m F(x(t))\right]^{-1 / 2} \leq 1 .
$$

Thus, for $t_{0} \leq t \leq t_{1}$, we have

$$
\int_{x(t)}^{x\left(t_{1}\right)}\left[y^{2}(t)-2 m F(s)\right]^{-1 / 2} d s \leq t_{1}-t \leq t_{1}-t_{0} .
$$

Now, from (i), given $k>0$, there exists $\bar{x}$ with $0<\bar{x}<x\left(t_{1}\right)$ so that $\int_{\bar{x}}^{x\left(t_{1}\right)}[-2 m F(s)]^{-1 / 2} d s>k$. Also, there exists $\delta>0$ so that if $y^{2}(t)<\delta$ and $x>\bar{x}$ then $y^{2}(t)-2 m F(s) \leq-8 m F(s)$. Now as $x\left(t_{0}\right)=y\left(t_{0}\right)=0$, there exists $\bar{t}$ with $t_{0}<\bar{t}<t_{1}$ so that $0<x(\bar{t})<\bar{x}$ and $y^{2}(\bar{t})<\delta$. Then,

$$
\begin{aligned}
t_{1}-t_{0} & \geq \int_{x(\bar{t})}^{x\left(t_{1}\right)}\left[y^{2}(\bar{t})-2 m F(s)\right]^{-1 / 2} d s \\
& \geq \frac{1}{2} \int_{\bar{x}}^{x\left(t_{1}\right)}[-2 m F(s)]^{-1 / 2} d s \geq k / 2 .
\end{aligned}
$$

This is a contradiction, however, as $k$ is arbitrarily large.

The argument in the third quadrant is similar. That argument completes the proof.

The following result not only characterizes the oscillation situation for (2) when $f$ is decreasing for large $|x|$, but it also is a new result for unretarded systems. Notice also that the remaining theorems differ significantly from many results of previous investigators (cf. [11], [2], and [15]) in that $\tau(t)$ need not be bounded. A notable exception to this is found in the work of Eliason [10] whose specific goal was to obtain the results of the previous investigators of the problem with $f(x)=|x|^{\gamma} \operatorname{sgn} x$ without assuming $\tau(t)$ bounded. Also, most previous investigators required that $f$ be bounded strictly away from zero for $|x| \geq d>0$.

Convention. We shall say that (2) is oscillatory or that all solutions of (2) oscillate if for every solution $(x(t), y(t))$ of $(2), x(t)$ has arbitrarily large zeros.

In the following theorems we usually ask that $a(t)>0$. This can be replaced by the more cumbersome condition that $a(t) \geq 0$ and $a(t)$ is not eventually identically zero. Obvious modifications then must be made in the proofs. For example, in the proof of Theorem 6 one would write " $y^{\prime} \leq 0$ and $y^{\prime}$ is not identically zero." 
Theorem 6. Let $0 \leq \tau(t), t-\tau(t) \rightarrow \infty$ as $t \rightarrow \infty, a(t)>0, x f(x)>0$ if $x \neq 0$, and let $f$ be decreasing for $|x| \geq d>0$ for some $d$. Then all solutions of (2) are oscillatory if and only if

$$
\int_{t_{0}}^{\infty} a(t) f\left( \pm c\left(t-t_{0}-\tau(t)\right) \pm x_{0}\right) d t= \pm \infty
$$

for every $c>0$, for every $t_{0} \geq 0$, and for every $x_{0} \geq d$.

Proof. Since $f$ is bounded, it follows easily that all solutions are continuable in the future.

We first show that if the integral condition holds, then all solutions are oscillatory. To this end, we show that any solution in Quadrant I with $x>d$ intersects the $x$-axis. That is, we assume that $(x(t), y(t))$ is a nonoscillatory solution and there exists $t_{0} \geq 0$ such that $x(t)>d$ and $y(t)>0$ for all $t \geq t_{0}$.

Notice that if $x(t)>d$ for all $t \geq t_{0}$, then there exists $t_{1}>t_{0}$ such that $x(t-\tau(t))>d$ for all $t \geq t_{1}$. Hence, $y^{\prime}=-a(t) f(x(t-\tau(t)))<0$ for all $t \geq t_{1}$. Thus, if there exists $t_{2}>t_{1}$ such that $y\left(t_{2}\right) \leq 0$, then $y(t)$ be comes negative; and, as $x^{\prime}=y, x(t)$ has a zero past $t_{2}$. Thus the assumption that $x(t)>d$ for all $t \geq t_{0}$ implies that $y(t)>0$ for $t \geq t_{0}$ by renaming $t_{0}$ if necessary.

Now if $d$ is replaced by zero in the two above paragraphs, then the conclusions are still valid. We now observe that if $x(t)>0$ for all $t \geq t_{0}$, then there is a $t_{1}>t_{0}$ with $x(t)>d$ for all $t \geq t_{1}$. To see this, notice that $f$ bounded and the divergent integral hypothesis holding implies that $\int_{0}^{\infty} a(s) d s=+\infty$. Thus, if $0<\bar{x} \leq x(t-\tau(t)) \leq d$ for all $t \geq t_{0}$ and some $\bar{x}$, then $f(x(t-\tau(t))) \geq p>0$ for some $p$ and all $t \geq t_{0}$. Then

$$
y(t)=y\left(t_{0}\right)-\int_{t_{0}}^{t} a(s) f(x(s-\tau(s))) d s \rightarrow-\infty
$$

and so $x(t-\tau(t))$ must become larger thăn $d$.

Now $y(t)$ decreasing for $t \geq t_{0}$ implies that $x^{\prime}(t)=y(t) \leq y\left(t_{0}\right)$ and so $x(t)$ $\leq y_{0}\left(t-t_{0}\right)+x\left(t_{0}\right)$ or $x(t-\tau(t)) \leq y_{0}\left(t-\tau(t)-t_{0}\right)+x\left(t_{0}\right)$ for $t \geq t_{0}$. Since $f$ is decreasing for $x>d$, we have

$$
\begin{aligned}
y(t) & =y_{0}-\int_{t_{0}}^{t} a(s) f(x(s-\tau(s))) d s \\
& \leq y_{0}-\int_{t_{0}}^{t} a(s) f\left(y_{0}\left(s-\tau(s)-t_{0}\right)+x\left(t_{0}\right)\right) d s
\end{aligned}
$$

which tends to $-\infty$. This is a contradiction and so the solution enters Quadrant IV. But then $x^{\prime}=y$ and $y^{\prime}<0$ so $x(t)$ subsequently has another zero as was argued above. A similar argument may be given in the left half-plane to show that a solution cannot remain in that half-plane.

Now suppose that all solutions oscillate and that $f$ is decreasing for $x>d$, but there exists $t_{0} \geq 0, c>0$, and $x_{0} \geq d$ such that 


$$
\int_{t_{0}}^{\infty} a(t) f\left(c\left(t-\tau(t)-t_{0}\right)+x_{0}\right) d t<\infty .
$$

Consider a solution $(x(t), y(t))$ which has initial function $x(t)=K\left(t-t_{0}\right)+x_{0}$ and $y(t)=K$ where $K>c$ and to be determined. So long as $y(t)>c$, then $x^{\prime}=y>c$ implies $x(t) \geq c\left(t-t_{0}\right)+x_{0}$ which in turn implies that $x(t-\tau(t))$ $\geq c\left(t-\tau(t)-t_{0}\right)+x_{0}$. Then

$$
\begin{aligned}
y(t) & =y_{0}-\int_{t_{0}}^{t} a(s) f(x(s-\tau(s))) d s \\
& \geq y_{0}-\int_{t_{0}}^{t} a(s) f\left(c\left(s-\tau(s)-t_{0}\right)+x_{0}\right) d s .
\end{aligned}
$$

Let $\int_{t_{0}}^{\infty} a(s) f\left(c\left(s-\tau(s)-t_{0}\right)+x_{0}\right) d s=M$ and pick $y_{0}=K>c+M$. Then $y(t)>c$ for all $t \geq t_{0}$ and so $x(t)$ does not oscillate. Similar arguments in Quadrant III complete the proof.

Theorem 7. Let $f$ be increasing and let $m$ be a continuous function satisfying $m(t):[0, \infty) \rightarrow(0, \infty)$. Then there is a solution $(x(t), y(t))$ of $(2)$ satisfying $x(t)$ $\geq m(t)$ for all $t \geq t_{0}$ only if $\int_{t_{0}}^{\infty} a(s) f(m(s-\tau(s))) d s<\infty$.

Proof. Suppose that there exists $x(t) \geq m(t)$, but $\int_{t_{0}}^{\infty} a(s) f(m(s-\tau(s))) d s$ $=+\infty$. Then $f$ is increasing and so

$$
y^{\prime}=-a(t) f(x(t-\tau(t))) \leq-a(t) f(m(t-\tau(t)))
$$

for $t \geq t_{1}>t_{0}$ where $t_{1}$ is chosen so that $t-\tau(t) \geq t_{0}$ if $t \geq t_{1}$. Thus, $y(t)$ $\leq y\left(t_{1}\right)-\int_{t_{1}}^{t} a(s) f(m(s-\tau(s))) d s \rightarrow-\infty$. Thus, as $x^{\prime}=y$, it follows that $x(t)$ becomes negative, contradicting $x(t) \geq m(t)>0$. This completes the proof.

We note that in the special case of $f(x)=|x|^{\gamma} \operatorname{sgn} x$, then Theorem 8 is implied by a result of Eliason [10]. .

A common result in the study of (2) both with and without retardation is that unless solutions oscillate, then there is a solution with $x(t)$ approximately $t^{\alpha}$ for $0<\alpha<1$. Theorem 7 provides a simple device for investigating the existence of such solutions. The result is also useful in connection with our next proposition.

Theorem 8. Let $a(t)>0$, let $f$ be increasing, and let all solutions of (2) be continuable to $+\infty$. For every solution $(x(t), y(t))$ of $(2), x(t)$ oscillates or $y(t) \rightarrow 0$ as $t \rightarrow \infty$ if and only if

$$
\int_{t_{0}}^{\infty} a(t) f\left( \pm c\left(t-\tau(t)-t_{0}\right) \pm x_{0}\right) d t= \pm \infty
$$

for every $c>0$, for every $t_{0} \geq 0$, and for every $x_{0} \geq 0$.

Proof. Assume that the integral condition holds. Let $(x(t), y(t))$ be a solution of (2) which remains in Quadrant I for all $t \geq t_{0}$ and assume that $y(t)$ does not tend 
to zero as $t$ tends to infinity. (That is, if $x(t)$ does not oscillate, then $(x(t), y(t))$ must eventually enter and remain in either Quadrant I or III. We deal with Quadrant I only, as Quadrant III is similar.) Then there exist $c>0, t_{0} \geq 0$, and $x_{0} \geq 0$ such that $y(t) \geq c, x(t-\tau(t))>0$ for all $t \geq t_{0}$. Thus, $x^{\prime}(t)=y(t) \geq c$ implies that $x(t) \geq x\left(t_{0}\right)+c\left(t-t_{0}\right)$ so $x(t-\tau(t)) \geq x\left(t_{0}\right)+c\left(t-\tau(t)-t_{0}\right)$ for $t-\tau(t) \geq t_{0}$ and so

$$
y^{\prime} \leq-a(t) f\left(x\left(t_{0}\right)+c\left(t-\tau(t)-t_{0}\right)\right)
$$

Let $t_{1} \geq t_{0}$ be chosen so that $t \geq t_{1}$ implies $t-\tau(t) \geq t_{0}$ and integrate from $t_{1}$ to $t$ yielding $y(t) \rightarrow-\infty$. This is a contradiction.

Now suppose that for every solution $(x(t), y(t))$, either $x(t)$ oscillates or $y(t) \rightarrow 0$ as $t \rightarrow \infty$. Assume also that there exist $t_{0} \geq 0, x_{0} \geq 0$, and $c>0$ such that

$$
\int_{t_{0}}^{\infty} a(t) f\left(c\left(t-\tau(t)-t_{0}\right)+x_{0}\right) d t=H<\infty .
$$

Start a solution $(x(t), y(t))$ having initial function $x(t)=c\left(t-t_{1}\right)+x_{1}$ and $y(t)=c$ on $E_{t_{1}}$ where $t_{0}<t_{1} \leq t$. Then $x^{\prime}(t)=y(t) \leq c$ implies that $x(t)$ $\leq c\left(t-t_{1}\right)+x_{1}$ and $x(t-\tau(t)) \leq c\left(t-\tau(t)-t_{1}\right)+x_{1}$ holds for $t \geq t_{1}$ because of the initial function. Hence,

$$
\begin{aligned}
y(t) & =y\left(t_{1}\right)-\int_{t_{1}}^{t} a(s) f(x(s-\tau(s))) d s \\
& \geq y\left(t_{1}\right)-\int_{t_{1}}^{t} a(s) f\left(c\left(s-\tau(s)-t_{1}\right)+x_{1}\right) d s .
\end{aligned}
$$

Now choose $x_{1}$ such that $-c t_{1}+x_{1}=-c t_{0}+x_{0}$ and choose $t_{1}$ so large that

$$
\int_{t_{1}}^{\infty} a(s) f\left(c\left(s-\tau(s)-t_{0}\right)+x_{0}\right) d s<c / 2 .
$$

Then $y(t)>c / 2$ for all $t \geq t_{1}$. This completes the proof.

There is no hope of deriving a simple divergence integral condition as a characterization of oscillations in general since for different positive values of $k$ in $x^{\prime \prime}(t)+\left[k / \tau^{2}\right] x(t)=0$ one has oscillation and nonoscillation. However, if $a(t)$ increases "most of the time", then such a condition can be formulated [4]. The following theorem is an extension of that result.

For $a^{\prime}(t)$ continuous and $a(t)$ positive, define $a_{+}^{\prime}(t)=\max \left[0, a^{\prime}(t)\right]$ and $a_{-}^{\prime}(t)$ $=\max \left[0,-a^{\prime}(t)\right]$ so that for $c(t)=a(0) \exp \int_{0}^{t}\left[a_{+}^{\prime}(\mu) / a(\mu)\right] d \mu$ and $b(t)$ $=\exp -\int_{0}^{t}\left[a_{-}^{\prime}(\mu) / a(\mu)\right] d \mu$ we have $a(t)=b(t) c(t)$.

Theorem 9. Let $a(t)>0, a^{\prime}(t)$ continuous, $\tau(t)$ continuously differentiable, and let $0<M_{1} \leq 1-\tau^{\prime}(t) \leq M_{2}$ for some constants $M_{1}$ and $M_{2}$ and all $t \geq 0$. Suppose also that for $a(t)=b(t) c(t)$ we have $b(t) \rightarrow b_{1}>0$. Then every continuable solution of (2) is oscillatory if and only if $\int_{t_{0}}^{\infty} a(t) f\left( \pm k\left(t-t_{0}\right)\right) d t= \pm \infty$ for every $k>0$ and every $t_{0} \geq 0$. 
Proof. Suppose $(x(t), y(t))$ is a solution of (2) for which $x(t)$ does not oscillate. Then if $x(t)>0$ for all $t \geq t_{0} \geq 0$, we must have $x^{\prime}(t)>0$ for $t \geq t_{1}$ for some $t_{1} \geq t_{0}$; otherwise, we would have $x^{\prime}(t) \leq 0$ and, as $x^{\prime \prime}(t)=-a(t) f(x(t-\tau(t)))$ $<0$ as long as $x(t-\tau(t))>0$, we see that $x(t)$ would become negative in finite time. Thus we see that $(x(t), y(t))$ remains in the first quadrant for $t \geq t_{1}$.

Now, as $x^{\prime}=y$ is nonincreasing for $t \geq t_{1}$, we have $x^{\prime}(t)=y(t) \leq y\left(t_{1}\right)=k_{1}$ and as $t-\tau(t) \rightarrow \infty$, there exists $t_{2}$ such that $t-\tau(t) \geq t_{1}$ for $t \geq t_{2}$. Thus, for $t \geq t_{2}$

$$
\begin{aligned}
{[x(t-\tau(t))]^{\prime} } & =x^{\prime}(t-\tau(t))\left(1-\tau^{\prime}(t)\right) \\
& =y(t-\tau(t))\left(1-\tau^{\prime}(t)\right) \leq k_{1} M_{2}=k .
\end{aligned}
$$

Letting $g(t)=x(t-\tau(t))$, we see that $g(t)$ is increasing for $t \geq t_{2}$, and that $g^{\prime}(t) \leq k$. Thus, $g(t)$ has an inverse $g^{-1}(s)$ and as $x(t-\tau(t))-x\left(t_{2}-\tau\left(t_{2}\right)\right)$ $=g(t)-g\left(t_{2}\right) \leq k\left(t-t_{2}\right)$ or, for $t \geq t_{2}$,

$$
g(t)=x(t-\tau(t)) \leq k\left(t-t_{2}+\frac{x\left(t_{2}-\tau\left(t_{2}\right)\right)}{k}\right)=k\left(t+M_{3}\right)
$$

where $M_{3}$ is a constant, we see that $g^{-1}(s) \geq[1 / k] s-M_{3}$.

Now if $x(t) \rightarrow \infty$ as $t \rightarrow \infty$, then $[x(t-\tau(t)) / k]-M_{3}$ will be nonnegative for $t \geq t_{3} \geq t_{2}$ for some $t_{3}$. From (2), we see that $y^{\prime}(t) / b(t)=-c(t) f(x(t-\tau(t)))$. As $y^{\prime}(t) \leq 0$ and $b(t) \geq b_{1}>0, y^{\prime}(t) / b_{1} \leq-c(t) f(x(t-\tau(t)))$ for $t \geq t_{3}$. Thus, for $t \geq t_{3}$

$$
\begin{aligned}
y(t) / b_{1} & \leq y\left(t_{3}\right) / b_{1}-\int_{t_{3}}^{t} c(\mu) f(g(\mu)) d \mu \\
& \leq y\left(t_{3}\right) / b_{1}-[1 / k] \int_{t_{3}}^{t} c(\mu) f(g(\mu)) g^{\prime}(\mu) d \mu \\
& =y\left(t_{3}\right) / b_{1}-[1 / k] \int_{g\left(t_{3}\right)}^{g(t)} c\left(g^{-1}(s)\right) f(s) d s .
\end{aligned}
$$

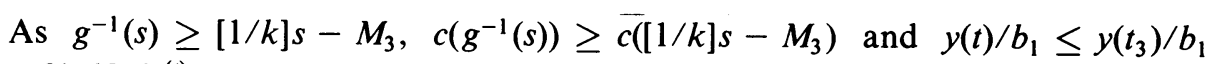

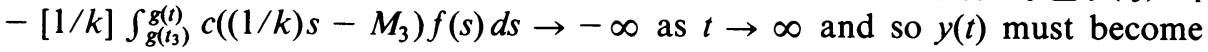
negative and we have a contradiction.

If $x(t)$ is bounded, then $x(t-\tau(t))$ tends to a positive limit $r$ and $y^{\prime}(t)$ $=-a(t) f(x(t-\tau(t))) \leq-a(t) f(r) / 2$ for $t$ sufficiently large. Thus, again we have $y(t)$ becoming negative. The argument if $x(t)$ is eventually negative is essentially the same and is omitted.

We now suppose that $\int_{t_{0}}^{\infty} a(t) f\left(k\left(t-t_{0}\right)\right) d t<\infty$ for some $k>0$ and some $t_{0}$. Then $\int_{t_{0}}^{\infty} a\left([1 / k] s+t_{0}\right) f(s) d s<\infty$ and, as $a(t)=b(t) c(t)$ we have

$$
\int_{t_{0}}^{\infty} c\left([1 / k] s+t_{0}\right) f(s) d s=M_{4}<\infty .
$$

Now if $k_{1}$ and $N$ are any constants with $k_{1}>k$, then $[1 / k] s+t_{0}>\left[1 / k_{1}\right] s+$ $t_{0}+N$ for $s$ sufficiently large. Thus, as $c(t)$ is nondecreasing, 


$$
\int_{t_{0}}^{\infty} c\left(\left[1 / k_{1}\right] s+t_{0}+N\right) f(s) d s<\infty
$$

In particular, for $k_{1} M_{1}=k_{2}>k$, we have $\int_{0}^{\infty} c\left(\left[1 / k_{1}\right] s+t^{*}\right) f(s) d s=M_{5}<\infty$ where $t^{*}-\tau\left(t^{*}\right)=0$.

Now, let $\left(x_{I}(t), y_{I}(t)\right)$ be an initial condition of (2) defined on $\left[t_{0}-\tau\left(t_{0}\right), 0\right]$, where $t_{0}-\tau\left(t_{0}\right)=\inf \{t-\tau(t): t \geq 0\}$, defined by $x_{I}(t) \equiv 0$ and $y_{I}(t)=A>k_{1}$ $>k$, where $A$ is to be determined. We see that for $t \geq 0, x(t)$ must become positive as $y(t)$ is continuous for $t \geq t_{0}-\tau\left(t_{0}\right)$ and so must remain positive on some interval $[0, w), w>0$. Thus, as long as $y(t)>0, x(t)$ is increasing and $y^{\prime}(t) \leq 0$ so $y(t)$ is nonincreasing. Also, we see that $x^{\prime}(t)=A$ on $\left[0, t^{*}\right]$ where $t^{*}-\tau\left(t^{*}\right)=0$.

As long as $y(t)>k_{1}>0$, for $t \geq t^{*}$, we have

$$
(x(t-\tau(t)))^{\prime}=y(t-\tau(t))\left(1-\tau^{\prime}(t)\right) \geq k_{1} M_{1}=k_{2}>0
$$

and so $x(t-\tau(t)) \geq k_{2}\left(t-t^{*}\right)$ for $t \geq t^{*}$. Letting $g(t)=x(t-\tau(t))$ as before, we see then that $g^{-1}(s) \leq\left[1 / k_{2}\right] s+t^{*}$.

From (2), it follows that $y(t) \geq y\left(t^{*}\right)-\int_{t^{*}}^{t} c(\mu) f(g(\mu)) d \mu$ and, as long as $y(t)>k_{1}$, we have

$$
\begin{aligned}
y(t) & \geq y\left(t^{*}\right)-\left[1 / k_{2}\right] \int_{t^{*}}^{t} c(\mu) f(g(\mu)) g^{\prime}(\mu) d \mu \\
& =A-\left[1 / k_{2}\right] \int_{g\left(t^{*}\right)}^{g(t)} c\left(g^{-1}(s)\right) f(s) d s \\
& \geq A-\left[1 / k_{2}\right] \int_{0}^{\infty} c\left(\left[1 / k_{2}\right] s+t^{*}\right) f(s) d s
\end{aligned}
$$

Now if $A$ is chosen so that $A-M_{5} / k_{2}>k_{1}$, we see that $y(t)$ remains larger than $k_{1}$ and so $x^{\prime}(t)=y(t)>k_{1}$ for all $t \geq 0$. Thus, $x(t) \geq k_{1} t$ and $x(t)$ does not oscillate. This completes the proof.

For notational purposes, it is now more convenient to write (2) as

$$
x^{\prime}(t)=y(t), \quad y^{\prime}(t)=-a(t) f(x(q(t)))
$$

in which $q:[0, \infty) \rightarrow R, f$ is nondecreasing, $q(t) \leq t$ for all $t, q(t) \rightarrow \infty$ as $t \rightarrow \infty, a(t)>0$, and $x f(x)>0$ if $x \neq 0$. Equation (3) is exactly the same as (2) with $t-\tau(t)=q(t)$.

Theorem 10. Suppose there exists $N$ such that for any sequence $t_{0}<t_{1}<\cdots$ $<t_{N+1}$ and any constants $c_{1}, \ldots, c_{N+2}$ with $c_{2}>0$ we have

$$
\begin{aligned}
& \int_{t_{N+1}}^{\infty} a(w) f\left(c_{N+2}+\int_{t_{N}}^{q(w)} s a(s) f\left(\ldots f\left(c_{1} \pm c_{2} \int_{t_{0}}^{q(v)} r a(r) d r\right) \ldots\right)\right) d w \\
& = \pm \infty
\end{aligned}
$$


Then all continuable solutions of (3) are oscillatory.

Proof. Suppose that $(x(t), y(t))$ is a solution of (3) such that for $t \geq t_{0}$ we have $y(t)>0$ and $x(q(t))>0$. It follows from (3) that

$$
x(t)=x\left(t_{0}\right)+x^{\prime}\left(t_{0}\right)\left(t-t_{0}\right)-\int_{t_{0}}^{t}(t-s) a(s) f(x(q(s))) d s
$$

and

$$
x^{\prime}(t)=x^{\prime}\left(t_{0}\right)-\int_{t_{0}}^{t} a(s) f(x(q(s))) d s
$$

Now

$$
\begin{aligned}
x(t) & =x\left(t_{0}\right)-x^{\prime}\left(t_{0}\right) t_{0}+t x^{\prime}(t)+\int_{t_{0}}^{t} s a(s) f(x(q(s))) d s \\
& \geq x\left(t_{0}\right)-x^{\prime}\left(t_{0}\right) t_{0}+\int_{t_{0}}^{t} s a(s) f(x(q(s))) d s \\
& \geq x\left(t_{0}\right)-x^{\prime}\left(t_{0}\right) t_{0}+\int_{t_{0}}^{t} s a(s) f\left(x\left(q\left(t_{0}\right)\right)\right) d s \\
& =c_{1}+c_{2} \int_{t_{0}}^{t} s a(s) d s
\end{aligned}
$$

for $t \geq t_{0}$. Thus, if $q(t) \geq t_{0}$, we have

$$
x(q(t)) \geq c_{1}+c_{2} \int_{t_{0}}^{q(t)} s a(s) d s .
$$

Now suppose $q(t) \geq t_{0}$ for $t \geq t_{1}$. Then for $t \geq t_{1}$, as before, we have

$$
x(t) \geq x\left(t_{1}\right)-x^{\prime}\left(t_{1}\right) t_{1}+\int_{t_{1}}^{t} s a(s) f(x(q(s))) d s
$$

and as $f$ is nondecreasing,

$$
x(t) \geq x\left(t_{1}\right)-x^{\prime}\left(t_{1}\right) t_{1}+\int_{t_{1}}^{t} s a(s) f\left(c_{1}+c_{2} \int_{t_{0}}^{q(s)} v a(v) d v\right) d s
$$

for $t \geq t_{1}$. Thus, if $q(t) \geq t_{1}$, we have

$$
x(q(t)) \geq c_{3}+\int_{t_{1}}^{q(t)} s a(s) f\left(c_{1}+c_{2} \int_{t_{0}}^{q(s)} \mu a(\mu) d \mu\right) d s
$$

where $c_{3}=x\left(t_{1}\right)-x^{\prime}\left(t_{1}\right) t_{1}$.

If $q(t) \geq t_{1}$, then for $t \geq t_{2}$ we obtain

$$
\begin{aligned}
x(t) \geq & x\left(t_{2}\right)-x^{\prime}\left(t_{2}\right) t_{2} \\
& +\int_{t_{2}}^{t} s a(s) f\left(c_{3}+\int_{t_{1}}^{q(s)} \mu a(\mu) f\left(c_{1}+c_{2} \int_{t_{0}}^{q(\mu)} v a(v) d v\right) d \mu\right) d s
\end{aligned}
$$


and

$$
x(q(t)) \geq c_{4}+\int_{t_{2}}^{q(t)} s a(s) f\left(c_{3}+\int_{t_{1}}^{q(s)} \mu a(\mu) f\left(c_{1}+c_{2} \int_{t_{0}}^{q(\mu)} v a(v) d v\right) d \mu\right) d s
$$

In general, there exists a sequence $t_{0}<t_{1}<\ldots<t_{N}$ such that for $t$ sufficiently large we have

$$
\begin{aligned}
x(q(t)) \geq c_{N+2}+\int_{t_{N}}^{q(t)}\left\{s a ( s ) f \left(c_{N+1}\right.\right. & +\int_{t_{N-1}}^{q(s)} \mu a(\mu) \\
& \left.\left.\cdot f\left(\ldots f\left(c_{1}+c_{2} \int_{t_{0}}^{q(\mu)} v a(v) d v\right) \ldots\right)\right) d s\right\}
\end{aligned}
$$

for $t \geq t_{N+1}$ where $q(t) \geq t_{N}$ for $t \geq t_{N+1}$. Now, as $y^{\prime}=-a(t) f(x(q(s)))$, integrating from $t_{N+1}$ to $t>t_{N+1}$, we have

$$
\begin{aligned}
y(t)-y\left(t_{N+1}\right) \leq-\int_{t_{N+1}}^{t}\{ & a(r) f\left(c_{N+2}\right. \\
& \left.\left.+\int_{t_{N}}^{q(r)} s a(s) f\left(\ldots f\left(c_{1}+c_{2} \int_{t_{0}}^{q(\mu)} v a(v) d v\right) \ldots\right)\right) d r\right\}
\end{aligned}
$$

and hence $y(t) \rightarrow-\infty$. This is a contradiction and so $x(t)$ must have a zero after $t_{0}$. The case for $x(t)$ negative is similar.

Example 1. Bradley [2] considered the linear delay equation

$$
x^{\prime \prime}(t)+a(t) x(t-\tau(t))=0
$$

where $a(t)$ and $\tau(t)$ are continuous on $[0, \infty)$ with $a(t) \geq 0$ and $0 \leq \tau(t) \leq M$ $<\infty$. He showed that if $\int_{0}^{\infty} a(t) d t=\infty$, then all solutions of (4) are oscillatory. From Theorem 10 we see that (4) is oscillatory if

$$
\int_{0}^{\infty} a(t)\left(c_{1}+c_{2} \int_{t_{0}}^{t-\tau(t)} s a(s) d s\right) d t=\infty
$$

for all $c_{1}$ and all $c_{2}>0$. Now, if $\int_{0}^{\infty} a(t) d t=\infty$, we see that $c_{1}+c_{2} \int_{t_{0}}^{t-\tau(t)} s a(s) d s$ is eventually greater than one and so $\int_{0}^{\infty} a(t)\left(c_{1}+c_{2} \int_{t_{0}}^{t-r(t)} s a(s) d s\right) d t=\infty$. Hence, Theorem 10 includes Bradley's result.

Example 2. Consider the equation

$$
x^{\prime \prime}+t^{-(1+\gamma)} x^{\sigma}\left(t^{\beta}\right)=0
$$

in which $\sigma$ is an odd integer, $0<\gamma<1$, and $0<\beta<1$.

Suppose there exists $N$ such that

$$
\int_{t_{N+1}}^{\infty} \mu^{-(1+\gamma)}\left(c_{N+2}+\int_{t_{N}}^{\mu^{\beta}} s^{-\gamma}(\ldots)^{\sigma}\right)^{\sigma} d \mu=\infty .
$$


Then for $N=0$ we have

$$
\begin{aligned}
\int_{t_{1}}^{\infty} & t^{-(1+\gamma)}\left(c_{1}+c_{2} \int_{t_{0}}^{t^{\beta}} s^{-\gamma} d s\right)^{\sigma} d t \\
& =\int_{t_{1}}^{\infty} t^{-(1+\gamma)}\left(c_{1}^{\prime}+c_{2}^{\prime} t^{(1-\gamma) \beta}\right)^{\sigma} d t \\
& =\int_{t_{1}}^{\infty}\left[c_{2}^{\prime} t^{-(1+\gamma)} t^{(1-\gamma) \beta \sigma}+\text { lower order term }\right] d t=\infty
\end{aligned}
$$

if $\beta \sigma \geq \gamma /(1-\gamma)$.

If $N=1$, then

$$
\begin{aligned}
\int_{t_{2}}^{\infty} t^{-(1+\gamma)}\left(c_{3}+\int_{t_{1}}^{t^{\beta}} s^{-\gamma}\left(c_{1}+c_{2} \int_{t_{0}}^{s^{\beta}} \mu^{-\gamma} d \mu\right)^{\sigma} d s\right)^{\sigma} d t \\
=\int_{t_{2}}^{\infty}\left[c_{2}^{\prime \prime \prime} t^{-(1+\gamma)+(1-\gamma) \beta_{\sigma}+(1-\gamma) \beta^{2} \sigma^{2}}+\text { lower order terms }\right] d t=\infty
\end{aligned}
$$

if $\beta \sigma+\beta^{2} \sigma^{2} \geq \gamma /(1-\gamma)$.

Generally, if $\sum_{j=1}^{N+1}(\beta \sigma)^{j} \geq \gamma /(1-\gamma)$, then solutions oscillate. But this is true if and only if $\sum_{j=1}^{\infty}(\beta \sigma)^{j}>\gamma /(1-\gamma)=\sum_{j=1}^{\infty} \gamma^{j}$ which is true if and only if $\beta \sigma>\gamma$. Thus, solutions oscillate if $\beta \sigma>\gamma$.

On the other hand, if $\beta \sigma<\gamma$, then

$$
\begin{aligned}
\int_{t_{0}}^{\infty} a(t) f(c(t-\tau(t))) d t & =\int_{t_{0}}^{\infty} t^{-(1+\gamma)}\left(c t^{\beta}\right)^{\sigma} d t \\
& =c_{1} \int_{t_{0}}^{\infty} t^{-(1+\gamma)+\beta o} d t<\infty
\end{aligned}
$$

which implies that there exist nonoscillatory solutions by Theorem 8 .

We note that our results differ from those previously obtained in that conditions to guarantee oscillation explicitly invoke $a(t), f(x)$, and $q(t)=t-\tau(t)$. Our next theorem shows that even in the case where $f(x)=x^{\gamma}$ it is often necessary to consider $a, f$, and $q$.

Theorem 11. Suppose $a(t) \geq 0$, not eventually identically zero, and $f$ is increasing in $x$. If $\int_{0}^{\infty} a(t) d t<\infty$, then there exists a continuous $q(t)$ defined for all $t \geq 0$ with $q(t) \leq t$ and $q(t) \rightarrow \infty$ such that (3) has nonoscillatory solutions.

Proof. We first assume that $f$ is not bounded above or below. Suppose $\int_{0}^{\infty} a(t) d t=M<\infty$ and let $\left\{t_{n}\right\}$ be the sequence tending to infinity with the property that $0=t_{0}<t_{1}<\ldots$ and $\int_{t_{n}}^{t_{n+1}} a(t) d t=(M / e)(1 / n !)$. Define $p_{1}(t)$ by $p_{1}(t)=2^{n}$ for $t_{n}<t<t_{n+1}$. We see then that

$$
\int_{0}^{\infty} a(t) p_{1}(t) d t=\sum_{n=0}^{\infty} \int_{t_{n}}^{t_{n+1}} a(t) p_{1}(t) d t=M e
$$


Now, define $p_{2}(t)$ by $p_{2}(t)=1$ for $t_{0} \leq t \leq t_{1}, p_{2}(t)=2^{n}$ for $\left(t_{n}+t_{n+1}\right) / 2 \leq t$ $\leq t_{n+1}$, and define it to be linear and continuous between $t_{n}$ and $\left(t_{n}+t_{n+1}\right) / 2$. If we define $q(t)$ by $q(t)=\min \left\{t, f^{-1}\left(p_{2}(t)\right)\right\}$, then $q(t)$ is continuous, $q(t) \leq t$, and $q(t) \rightarrow \infty$ as $t \rightarrow \infty$. We now have

$$
\begin{aligned}
\int_{0}^{\infty} a(t) f(q(t)) d t & \leq \int_{0}^{\infty} a(t) p_{2}(t) d t \\
& \leq \int_{0}^{\infty} a(t) p_{1}(t) d t<\infty
\end{aligned}
$$

It now follows from Theorem 8 that (3) has a nonoscillatory solution.

If $f$ is bounded above or below, then the existence of a nonoscillatory solution follows from Theorem 8 with any $q(t)$. This completes the proof.

We note that Theorem 11 shows that the result obtained by Waltman [16] is the best possible result under the conditions given. In fact, combining Waltman's result with our Theorem 11 yields the following result.

Corollary. Suppose $a(t) \geq 0$, not eventually identically zero, and $f$ is increasing in $x$. A necessary and sufficient condition that (3) is oscillatory for every choice of $q(t)$ with $q(t) \leq t$ and $q(t) \rightarrow \infty$ as $t \rightarrow \infty$ is $\int_{0}^{\infty} a(t) d t=\infty$.

\section{REFERENCES}

1. F. V. Atkinson, On second-order nonlinear oscillations, Pacific J. Math. 5 (1955), 643-647. MR 17, 264.

2. J. S. Bradley, Oscillation theorems for a second-order delay equation, J. Differential Equations 8 (1970), 397-403. MR 42 \#3379.

3. T. Burton and R. Grimmer, Stability properties of $\left(r(t) u^{\prime}\right)^{\prime}+a(t) f(u) g\left(u^{\prime}\right)=0$, Monatsh. Math. 74 (1970), 211-222.

4. - On the asymptotic behavior of solutions of $x^{\prime \prime}+a(t) f(x)=0$, Proc. Cambridge Philos. Soc. 70 (1971), 77-88.

5.—-On continuability of solutions of second order differential equations, Proc. Amer. Math. Soc. 29 (1971), 277-283. MR 43 \#3541.

6. - On the asymptotic behavior of solutions of $x^{\prime \prime}+a(t) f(x)=e(t)$, Pacific J. Math. 41 (1972), 43-55.

7. D. W. Bushaw, The differential equation $x^{\prime \prime}+g\left(x, x^{\prime}\right)+h(x)=e(t)$, Terminal Report on Contract AF 29(600)-1003, Holloman Air Force Base, New Mexico, 1958.

8. E. A. Coddington and N. Levinson, Theory of ordinary differential equations, McGraw-Hill, New York, 1955, p. 61. MR 16, 1022.

9. R. D. Driver, Existence theory for a delay-differential system, Contributions to Differential Equations 1 (1963), 317-366. MR 27 \#420.

10. S. B. Eliason, Nonoscillation theorems for certain second order functional differential equations, Unpublished and undated preprint, University of Oklahoma, Norman, Oklahoma.

11. H. E. Gollwitzer, On nonlinear oscillations for a second order delay equation, J. Math. Anal. Appl. 26 (1969), 385-389. MR 39 \#581.

12. J. K. Hale, Ordinary differential equations, Wiley, New York, 1969, p. 33. 
13. S. P. Hastings, Boundary value problems in one differential equation with a discontinuity, $\mathrm{J}$. Differential Equations 1 (1965), 346-369. MR 31 \#4954.

14. M. N. Oğuztöreli, Time-lag systems, Math. in Sci. and Engineering, vol. 24, Academic Press, New York, 1966. MR 36 \#484.

15. V. A. Staikos and A. G. Petsoulas, Some oscillation criteria for second order nonlinear delaydifferential equations, J. Math. Anal. Appl. 30 (1970), 695-701. MR 41 \#2171.

16. Paul Waltman, $A$ note on an oscillation criterion for an equation with a functional argument, Canad. Math. Bull. 11 (1968), 593-595. MR 38 \#6193.

Department of Mathematics, Southern Illinois University, Carbondale, Illinois 62901 